\title{
Generalized Electron Balance for Dynamic Redox Systems in Mixed-Solvent Media
}

\author{
Anna Maria Michałowska-Kaczmarczyk¹, Tadeusz Michałowski2* \\ ${ }^{1}$ Department of Oncology, The University Hospital in Cracow, Cracow, Poland \\ ${ }^{2}$ Faculty of Engineering and Chemical Technology, Cracow University of Technology, Cracow, Poland \\ Email: michalot@o2.pl
}

Received 16 September 2014; revised 25 October 2014; accepted 5 November 2014

Copyright (C) 2014 by authors and Scientific Research Publishing Inc.

This work is licensed under the Creative Commons Attribution International License (CC BY).

http://creativecommons.org/licenses/by/4.0/

(c) (i) Open Access

\begin{abstract}
A complex example of electrolytic redox system involving 47 species, 3 electron-active elements and five ( 3 amphiprotic +2 aprotic) co-solvents, is presented. Mixed solvates of the species thus formed are admitted in the system considered. It is proved that the Generalized Electron Balance (GEB) in its simplest form obtained according to the Approach II to GEB is identical with the one obtained for aqueous media and binary-solvent system, and is equivalent to the Approach I to GEB.
\end{abstract}

\section{Keywords}

Electrolytic Redox Systems, Generalized Electron Balance, Mixed-Solvent Media

\section{Introduction}

Motto: "Everything should be made as simple as possible, but not simpler” [1].

In the previous issues [2]-[8] and in earlier papers cited therein, the concept of Generalized Electron Balance (GEB), completing the set of compatible equations necessary for quantitative/mathematical solution of electrolytic redox systems, was introduced as two alternative options, named as Approach I and Approach II to GEB. In both Approaches it is assumed/admitted, that all the species $X_{i}^{z_{i}}$ exist in an electrolytic system in their natural form, i.e., as solvates. In particular, there are hydrates $X_{i}^{z_{i}} \cdot n_{i W}$ in aqueous $\left(\mathrm{W}=\mathrm{H}_{2} \mathrm{O}\right)$ media, $X_{i}^{z_{i}} \cdot n_{i W} n_{i A}$, $X_{i}^{z_{i}} \cdot n_{i W} n_{i B}$ or $X_{i}^{z_{i}} \cdot n_{i A} n_{i B}$ in binary-solvent media (W, A), (W, B) or (A, B) [9]-[11], respectively. The values of $n_{W i}, n_{A i}$ and $n_{B i}$, considered as mean numbers of $\mathrm{W}, \mathrm{A}$ and $\mathrm{B}$ attached to $X_{i}^{z_{i}}$, are unknown, in principle, and vary with the co-solvent(s) composition, and solute(s) concentration.

In this paper, we refer also to more complex media with the mixture of co-solvents: W, A, B, E and F. We assume that the co-solvents are mutually miscible and at least one of the co-solvents has amphiprotic properties *Corresponding author.

How to cite this paper: Michałowska-Kaczmarczyk, A.M. and Michałowski, T. (2014) Generalized Electron Balance for Dynamic Redox Systems in Mixed-Solvent Media. Journal of Analytical Sciences, Methods and Instrumentation, 4, $102-109$. http://dx.doi.org/10.4236/jasmi.2014.44014 
[5]. Eeach of the co-solvents has potential/real solvating properties, i.e., $n_{i W}, n_{i A}, n_{i B}, n_{i E}, n_{i F} \geq 0$ in the solvated species $X_{i}^{z_{i}} \cdot n_{i W} n_{i A} n_{i B} n_{i E} n_{i F}$. Let $X_{i}^{z_{i}}\left(N_{i}, n_{i W}, n_{i A}, n_{i B}, n_{i E}, n_{i F}\right)$ denotes $N_{i}$ entities of these species in the related system. In further part of the paper, we assume $\mathrm{W}=\mathrm{H}_{2} \mathrm{O}, \mathrm{A}=\mathrm{CH}_{3} \mathrm{OH}, \mathrm{B}=\mathrm{C}_{2} \mathrm{H}_{5} \mathrm{OH}, \mathrm{E}=\left(\mathrm{CH}_{3}\right)_{2} \mathrm{SO}, \mathrm{F}=\mathrm{CH}_{3} \mathrm{CN}$; W, A, B have amphiprotic properties, and E, F-have not. In particular, $N_{15}$ ions $\mathrm{H}_{4} \mathrm{IO}_{6}^{-} \cdot n_{15 W} n_{15 A} n_{15 B} n_{15 E} n_{15 F}$ contain: $N_{15}\left(4+4 n_{15 \mathrm{~A}}+6 n_{15 B}+6 n_{15 E}+3 n_{15 F}\right)$ atoms of $\mathrm{H}, N_{15}\left(6+n_{15 \mathrm{~W}}+n_{15 \mathrm{~A}}+n_{15 B}+n_{15 E}\right)$ atoms of O, $N_{15}$ atoms of I, $\quad N_{15}\left(n_{15 \mathrm{~A}}+2 n_{15 B}+2 n_{15 E}+2 n_{15 F}\right)$ atoms of C, $N_{15} n_{15 E}$ atoms of S, and $N_{15} n_{15 F}$ atoms of N. It is assumed that the solvents do not form-with solvates-the species $X_{i}^{z_{i}}$ other than those formed in (known from) aqueous media. In other words, W, A, B, E, F enter (potentially) the solvating sphere of $X_{i}^{z_{i}}$. On this basis, the elemental balances $f(E(\mathrm{i}))$ for particular elements $E(\mathrm{i})$ are formulated. For ordering purposes, we denote: $E(1)=$ $\mathrm{H}, E(2)=\mathrm{O}, E(3)=\mathrm{I}, E(4)=\mathrm{C}, E(5)=\mathrm{S}, E(6)=\mathrm{N}$. We apply also the balances $f(\boldsymbol{C}(\mathrm{Y}))$ for the cores $C(\mathrm{Y}), \mathrm{Y}=$ $\mathrm{A}, \mathrm{B}, \mathrm{E}, \mathrm{F}$. The "core" is a cluster of elements of the same composition, structure and charge, that does not undergo a change in the system in question; e.g., $\mathrm{CH}_{3} \mathrm{OH}, \mathrm{CH}_{3} \mathrm{OH}_{2}^{+}$and $\mathrm{CH}_{3} \mathrm{O}^{-}$contain the cluster $\mathrm{CH}_{3} \mathrm{O}^{-}$, considered as the core. We denote $\boldsymbol{C}(\mathrm{A})=\mathrm{CH}_{3} \mathrm{O}^{-}, \boldsymbol{C}(\mathrm{B})=\mathrm{C}_{2} \mathrm{H}_{5} \mathrm{O}^{-}, \boldsymbol{C}(\mathrm{E})=\left(\mathrm{CH}_{3}\right)_{2} \mathrm{SO}=\mathrm{E}, \boldsymbol{C}(\mathrm{F})=\mathrm{CH}_{5} \mathrm{CN}=\mathrm{F}$. The species: $\mathrm{H}_{2} \mathrm{CO}_{3}, \mathrm{HCO}_{3}^{-}$and $\mathrm{CO}_{3}^{2-}$ have $\mathrm{CO}_{3}^{2-}$ as the common core. However, $\mathrm{ClO}_{2}^{-}$(anion of $\mathrm{HClO}_{2}$ ) and $\mathrm{ClO}_{2}$ (chlorine dioxide) have different cores.

All the balances in this paper will be presented explicitly, to check the validity of the reasoning and accept it without reservations.

\section{Formulation of Balances}

Let us consider a system obtained after addition of $V \mathrm{~mL}$ of titrant $(\mathrm{T})$ containing $\mathrm{I}_{2}(\mathrm{C})+\mathrm{KI}\left(\mathrm{C}_{1}\right)+\mathrm{CO}_{2}\left(\mathrm{C}_{2}\right)$ in $\mathrm{A}+\mathrm{B}+\mathrm{E}+\mathrm{F}$ into $V_{0} \mathrm{~mL}$ of titrand (D) containing $\mathrm{KBrO}_{3}\left(\mathrm{C}_{0}\right)+\mathrm{HCl}\left(\mathrm{C}_{01}\right)+\mathrm{CO}_{2}\left(\mathrm{C}_{02}\right)$ in W + A + E; all concentrations are expressed in $\mathrm{mol} / \mathrm{L}$. The volume $V_{0} \mathrm{~mL}$ of $\mathrm{D}$ is composed of $N_{01}$ molecules of $\mathrm{KBrO}_{3}, N_{02}$ molecules of $\mathrm{HCl}, N_{03}$ molecules of $\mathrm{CO}_{2}, N_{04}$ molecules of W, $N_{05}$ molecules of A, and $N_{06}$ molecules of E; $\mathrm{V}$ mL of T is composed of $N_{07}$ molecules of $\mathrm{I}_{2}, N_{08}$ molecules of KI, $N_{09}$ molecules of $\mathrm{CO}_{2}$ and $N_{011}$ molecules of A, $N_{012}$ molecules of B, $N_{013}$ molecules of E, and $\mathrm{N}_{014}$ molecules of F.

We assume that the solutes composing $\mathrm{D}$ and $\mathrm{T}$ were introduced in single solvents or mixtures of solvents. In ca. $V_{0}+V \mathrm{~mL}$ of a $\mathrm{D}+\mathrm{T}$ mixture thus obtained, we have the following species (all changes in oxidation degrees are admitted).

$$
\begin{aligned}
& \mathrm{H}_{2} \mathrm{O}\left(N_{1}\right), \mathrm{H}^{+}\left(N_{2}, n_{2 W}, n_{2 A}, n_{2 B}, n_{2 E}, n_{2 F}\right), \mathrm{OH}^{-}\left(N_{3}, n_{3 W}, n_{3 A}, n_{3 B}, n_{3 E}, n_{3 F}\right), \\
& \mathrm{K}^{+}\left(N_{4}, n_{4 W}, n_{4 A}, n_{4 B}, n_{4 E}, n_{4 F}\right), \mathrm{I}^{-}\left(N_{5}, n_{5 W}, n_{5 A}, n_{5 B}, n_{5 E}, n_{5 F}\right), \\
& \mathrm{I}_{3}^{-}\left(N_{6}, n_{6 W}, n_{6 A}, n_{6 B}, n_{6 E}, n_{6 F}\right), \mathrm{I}_{2}\left(N_{7}, n_{7 W}, n_{7 A}, n_{7 B}, n_{7 E}, n_{7 F}\right), \\
& \mathrm{I}_{2(s)}\left(N_{8}, n_{8 W}, n_{8 A}, n_{8 B}, n_{8 E}, n_{8 F}\right), \mathrm{HIO}\left(N_{9}, n_{9 W}, n_{9 A}, n_{9 B}, n_{9 E}, n_{9 F}\right), \\
& \mathrm{IO}^{-}\left(N_{11}, n_{11 W}, n_{11 A}, n_{11 B}, n_{11 E}, n_{11 F}\right), \mathrm{HIO}\left(N_{12}, n_{12 W}, n_{12 A}, n_{12 B}, n_{12 E}, n_{12 F}\right), \\
& \mathrm{IO}_{3}^{-}\left(N_{13}, n_{13 W}, n_{13 A}, n_{13 B}, n_{13 E}, n_{13 F}\right), \mathrm{H}_{5} \mathrm{IO}_{6}\left(N_{14}, n_{14 W}, n_{14 A}, n_{14 B}, n_{14 E}, n_{14 F}\right), \\
& \mathrm{H}_{4} \mathrm{IO}_{6}^{-}\left(N_{15}, n_{15 W}, n_{15 A}, n_{15 B}, n_{15 E}, n_{15 F}\right), \mathrm{H}_{3} \mathrm{IO}_{6}^{2-}\left(N_{16}, n_{16 W}, n_{16 A}, n_{16 B}, n_{16 E}, n_{16 F}\right), \\
& \mathrm{Cl}^{-}\left(N_{17}, n_{17 W}, n_{17 A}, n_{17 B}, n_{17 E}, n_{17 F}\right), \mathrm{Cl}_{2}\left(N_{18}, n_{18 W}, n_{18 A}, n_{18 B}, n_{18 E}, n_{18 F}\right), \\
& \mathrm{HClO}\left(N_{19}, n_{19 W}, n_{19 A}, n_{19 B}, n_{19 E}, n_{19 F}\right), \mathrm{ClO}^{-}\left(N_{21}, n_{21 W}, n_{21 A}, n_{21 B}, n_{21 E}, n_{21 F}\right), \\
& \mathrm{HClO}_{2}\left(N_{22}, n_{22 W}, n_{22 A}, n_{22 B}, n_{22 E}, n_{22 F}\right), \mathrm{ClO}_{2}^{-}\left(N_{23}, n_{23 W}, n_{23 A}, n_{23 B}, n_{23 E}, n_{23 F}\right), \\
& \mathrm{ClO}_{2}\left(N_{24}, n_{24 W}, n_{24 A}, n_{24 B}, n_{24 E}, n_{24 F}\right), \mathrm{ClO}_{3}^{-}\left(N_{25}, n_{25 W}, n_{25 A}, n_{25 B}, n_{25 E}, n_{25 F}\right), \\
& \mathrm{ClO}_{4}^{-}\left(N_{26}, n_{26 W}, n_{26 A}, n_{26 B}, n_{26 E}, n_{26 F}\right), \mathrm{Br}^{-}\left(N_{27}, n_{27 W}, n_{27 A}, n_{27 B}, n_{27 E}, n_{27 F}\right),
\end{aligned}
$$


$\operatorname{Br}_{3}^{-}\left(N_{28}, n_{28 W}, n_{28 A}, n_{28 B}, n_{28 E}, n_{28 F}\right), \mathrm{Br}_{2}\left(N_{29}, n_{29 W}, n_{29 A}, n_{29 B}, n_{29 E}, n_{29 F}\right)$,

$\operatorname{HBrO}\left(N_{31}, n_{31 W}, n_{31 A}, n_{31 B}, n_{31 E}, n_{31 F}\right), \operatorname{BrO}^{-}\left(N_{32}, n_{32 W}, n_{32 A}, n_{32 B}, n_{32 E}, n_{32 F}\right)$,

$\mathrm{HBrO}_{3}\left(N_{33}, n_{33 W}, n_{33 A}, n_{33 B}, n_{33 E}, n_{33 F}\right), \mathrm{BrO}_{3}^{-}\left(N_{34}, n_{34 W}, n_{34 A}, n_{34 B}, n_{34 E}, n_{34 F}\right)$,

$\mathrm{I}_{2} \mathrm{Cl}^{-}\left(N_{35}, n_{35 W}, n_{35 A}, n_{35 B}, n_{35 E}, n_{35 F}\right), \operatorname{ICl}\left(N_{36}, n_{36 W}, n_{36 A}, n_{36 B}, n_{36 E}, n_{36 F}\right)$,

$\operatorname{ICl}_{2}^{-}\left(N_{37}, n_{37 W}, n_{37 A}, n_{37 B}, n_{37 E}, n_{37 F}\right), \operatorname{IBr}\left(N_{38}, n_{38 W}, n_{38 A}, n_{38 B}, n_{38 E}, n_{38 F}\right)$,

$\operatorname{IBr}_{2}^{-}\left(N_{39}, n_{39 W}, n_{39 A}, n_{39 B}, n_{39 E}, n_{39 F}\right), \mathrm{H}_{2} \mathrm{CO}_{3}\left(N_{41}, n_{41 W}, n_{41 A}, n_{41 B}, n_{41 E}, n_{41 F}\right)$,

$\mathrm{HCO}_{3}^{-}\left(N_{42}, n_{42 W}, n_{42 A}, n_{42 B}, n_{42 E}, n_{42 F}\right), \mathrm{CO}_{3}^{2-}\left(N_{43}, n_{43 W}, n_{43 A}, n_{43 B}, n_{43 E}, n_{43 F}\right)$,

$\mathrm{CH}_{3} \mathrm{OH}\left(N_{44}\right), \mathrm{CH}_{3} \mathrm{OH}_{2}^{+}\left(N_{45}, n_{45 W}, n_{45 A}, n_{45 B}, n_{45 E}, n_{45 F}\right), \mathrm{CH}_{3} \mathrm{O}^{-}\left(N_{46}, n_{46 W}, n_{46 A}, n_{46 B}, n_{46 E}, n_{46 F}\right)$,

$\mathrm{C}_{2} \mathrm{H}_{5} \mathrm{OH}\left(N_{47}\right), \mathrm{C}_{2} \mathrm{H}_{5} \mathrm{OH}_{2}^{+}\left(N_{48}, n_{48 W}, n_{48 A}, n_{48 B}, n_{48 E}, n_{48 F}\right), \mathrm{C}_{2} \mathrm{H}_{5} \mathrm{O}^{-}\left(N_{49}, n_{49 W}, n_{49 A}, n_{49 B}, n_{49 E}, n_{49 F}\right)$, $\left(\mathrm{CH}_{3}\right)_{2} \mathrm{SO}\left(N_{51}\right), \mathrm{CH}_{3} \mathrm{CN}\left(N_{52}\right)$

where $\mathrm{I}_{2(\mathrm{~s})}$ - solid iodine, $\mathrm{I}_{2}$ - soluble iodine. In the above list of species, $\mathrm{H}_{2} \mathrm{O}\left(N_{1}\right), \mathrm{CH}_{3} \mathrm{OH}\left(N_{44}\right), \mathrm{C}_{2} \mathrm{H}_{5} \mathrm{OH}\left(N_{47}\right)$, $\left(\mathrm{CH}_{3}\right)_{2} \mathrm{SO}\left(\mathrm{N}_{51}\right)$ and $\mathrm{CH}_{3} \mathrm{CN}\left(N_{52}\right)$ are free molecules of the corresponding solvents, i.e., not involved in the solvates. We prove that the numbers: $N_{1}, N_{44}, N_{47}, N_{51}$ and $N_{52}$ of the free molecules and the numbers: $n_{i W}, n_{i A}, n_{i B}$, $n_{i E}, n_{i F}$ of these molecules in the solvates do not enter the simplest form of the resulting GEB.

The elemental balances: $f(\mathrm{H})$ for $\mathrm{H}$, and $f(\mathrm{O})$ for $\mathrm{O}$ are as follows:

- $f(\mathrm{H})$

$$
\begin{aligned}
& 2 N_{1}+N_{2}\left(1+2 n_{2 W}+4 n_{2 A}+6 n_{2 B}+6 n_{2 E}+3 n_{2 F}\right)+N_{3}\left(1+2 n_{3 W}+4 n_{3 A}+6 n_{3 B}+6 n_{3 E}+3 n_{3 F}\right) \\
& +N_{4}\left(2 n_{4 W}+4 n_{4 A}+6 n_{4 B}+6 n_{4 E}+3 n_{4 F}\right)+N_{5}\left(2 n_{5 W}+4 n_{5 A}+6 n_{5 B}+6 n_{5 E}+3 n_{5 F}\right) \\
& +N_{6}\left(2 n_{6 W}+4 n_{6 A}+6 n_{6 B}+6 n_{6 E}+3 n_{6 F}\right)+N_{7}\left(2 n_{7 W}+4 n_{7 A}+6 n_{7 B}+6 n_{7 E}+3 n_{7 F}\right) \\
& +N_{8}\left(2 n_{8 W}+4 n_{8 A}+6 n_{8 B}+6 n_{8 E}+3 n_{8 F}\right)+N_{9}\left(1+2 n_{9 W}+4 n_{9 A}+6 n_{9 B}+6 n_{9 E}+3 n_{9 F}\right) \\
& +N_{11}\left(2 n_{11 W}+4 n_{11 A}+6 n_{11 B}+6 n_{11 E}+3 n_{11 F}\right)+N_{12}\left(1+2 n_{12 W}+4 n_{12 A}+6 n_{12 B}+6 n_{12 E}+3 n_{12 F}\right) \\
& +N_{13}\left(2 n_{13 W}+4 n_{13 A}+6 n_{13 B}+6 n_{13 E}+3 n_{13 F}\right)+N_{14}\left(5+2 n_{14 W}+4 n_{14 A}+6 n_{14 B}+6 n_{14 E}+3 n_{14 F}\right) \\
& +N_{15}\left(4+2 n_{15 W}+4 n_{15 A}+6 n_{15 B}+6 n_{15 E}+3 n_{15 F}\right)+N_{16}\left(3+2 n_{16 W}+4 n_{16 A}+6 n_{16 B}+6 n_{16 E}+3 n_{16 F}\right) \\
& +N_{17}\left(2 n_{17 W}+4 n_{17 A}+6 n_{17 B}+6 n_{17 E}+3 n_{17 F}\right)+N_{18}\left(2 n_{18 W}+4 n_{18 A}+6 n_{18 B}+6 n_{18 E}+3 n_{18 F}\right) \\
& +N_{19}\left(1+2 n_{19 W}+4 n_{19 A}+6 n_{19 B}+6 n_{19 E}+3 n_{19 F}\right)+N_{21}\left(2 n_{21 W}+4 n_{21 A}+6 n_{21 B}+6 n_{21 E}+3 n_{21 F}\right) \\
& +N_{22}\left(1+2 n_{22 W}+4 n_{22 A}+6 n_{22 B}+6 n_{22 E}+3 n_{22 F}\right)+N_{23}\left(2 n_{23 W}+4 n_{23 A}+6 n_{23 B}+6 n_{23 E}+3 n_{23 F}\right) \\
& +N_{24}\left(2 n_{24 W}+4 n_{24 A}+6 n_{24 B}+6 n_{24 E}+3 n_{24 F}\right)+N_{25}\left(2 n_{25 W}+4 n_{25 A}+6 n_{25 B}+6 n_{25 E}+3 n_{25 F}\right) \\
& +N_{26}\left(2 n_{26 \mathrm{~W}}+4 n_{26 \mathrm{~A}}+6 n_{26 \mathrm{~B}}+6 n_{26 \mathrm{E}}+3 n_{26 \mathrm{~F}}\right)+N_{27}\left(2 n_{27 \mathrm{~W}}+4 n_{27 \mathrm{~A}}+6 n_{27 B}+6 n_{27 E}+3 n_{27 F}\right) \\
& +N_{28}\left(2 n_{28 W}+4 n_{28 A}+6 n_{28 B}+6 n_{28 E}+3 n_{28 F}\right)+N_{29}\left(2 n_{29 W}+4 n_{29 A}+6 n_{29 B}+6 n_{29 E}+3 n_{29 F}\right) \\
& +N_{31}\left(1+2 n_{31 W}+4 n_{31 A}+6 n_{31 B}+6 n_{31 E}+3 n_{31 F}\right)+N_{32}\left(2 n_{32 W}+4 n_{32 A}+6 n_{32 B}+6 n_{32 E}+3 n_{32 F}\right) \\
& +N_{33}\left(1+2 n_{33 W}+4 n_{33 A}+6 n_{33 B}+6 n_{33 E}+3 n_{33 F}\right)+N_{34}\left(2 n_{34 W}+4 n_{34 A}+6 n_{34 B}+6 n_{34 E}+3 n_{34 F}\right) \\
& +N_{35}\left(2 n_{35 W}+4 n_{35 A}+6 n_{35 B}+6 n_{35 E}+3 n_{35 F}\right)+N_{36}\left(2 n_{36 W}+4 n_{36 A}+6 n_{36 B}+6 n_{36 E}+3 n_{36 F}\right) \\
& +N_{37}\left(2 n_{37 W}+4 n_{37 A}+6 n_{37 B}+6 n_{37 E}+3 n_{37 F}\right)+N_{38}\left(2 n_{38 W}+4 n_{38 A}+6 n_{38 B}+6 n_{38 E}+3 n_{38 F}\right) \\
& +N_{39}\left(2 n_{39 W}+4 n_{39 A}+6 n_{39 B}+6 n_{39 E}+3 n_{39 F}\right)+N_{41}\left(2+2 n_{41 W}+4 n_{41 A}+6 n_{41 B}+6 n_{41 E}+3 n_{41 F}\right) \\
& +N_{42}\left(1+2 n_{42 W}+4 n_{42 A}+6 n_{42 B}+6 n_{42 E}+3 n_{42 F}\right)+N_{43}\left(2 n_{43 W}+4 n_{43 A}+6 n_{43 B}+6 n_{43 E}+3 n_{43 F}\right)+4 N_{44} \\
& +N_{45}\left(5+2 n_{45 W}+4 n_{45 A}+6 n_{45 B}+6 n_{45 E}+3 n_{45 F}\right)+N_{46}\left(3+2 n_{46 W}+4 n_{46 A}+6 n_{46 B}+6 n_{46 E}+3 n_{46 F}\right)+6 N_{47} \\
& +N_{48}\left(7+2 n_{48 W}+4 n_{48 A}+6 n_{48 B}+6 n_{48 E}+3 n_{48 F}\right)+N_{49}\left(5+2 n_{49 W}+4 n_{49 A}+6 n_{49 B}+6 n_{49 E}+3 n_{49 F}\right) \\
& +6 N_{51}+3 N_{52}=N_{02}+2 N_{04}+4 N_{05}+6 N_{06}+4 N_{011}+6 N_{012}+6 N_{013}+3 N_{014}
\end{aligned}
$$


- $f(\mathrm{O})$

$$
\begin{aligned}
& N_{1}+N_{2}\left(n_{2 W}+n_{2 A}+n_{2 B}+n_{2 E}\right)+N_{3}\left(1+n_{3 W}+n_{3 A}+n_{3 B}+n_{3 E}\right)+N_{4}\left(n_{4 W}+n_{4 A}+n_{4 B}+n_{4 E}\right) \\
& +N_{5}\left(n_{5 W}+n_{5 A}+n_{5 B}+n_{5 E}\right)+N_{6}\left(n_{6 W}+n_{6 A}+n_{6 B}+n_{6 E}\right)+N_{7}\left(n_{7 W}+n_{7 A}+n_{7 B}+n_{7 E}\right) \\
& +N_{8}\left(n_{8 W}+n_{8 A}+n_{8 B}+n_{8 E}\right)+N_{9}\left(1+n_{9 W}+n_{9 A}+n_{9 B}+n_{9 E}\right)+N_{11}\left(1+n_{11 W}+n_{11 A}+n_{11 B}+n_{11 E}\right) \\
& +N_{12}\left(3+n_{12 W}+n_{12 A}+n_{12 B}+n_{12 E}\right)+N_{13}\left(3+n_{13 W}+n_{13 A}+n_{13 B}+n_{13 E}\right)+N_{14}\left(6+n_{14 W}+n_{14 A}+n_{14 B}+n_{14 E}\right) \\
& +N_{15}\left(6+n_{15 \mathrm{~W}}+n_{15 \mathrm{~A}}+n_{15 B}+n_{15 E}\right)+N_{16}\left(6+n_{16 \mathrm{~W}}+n_{16 \mathrm{~A}}+n_{16 \mathrm{~B}}+n_{16 E}\right)+N_{17}\left(n_{17 \mathrm{~W}}+n_{17 \mathrm{~A}}+n_{17 \mathrm{~B}}+n_{17 E}\right) \\
& +N_{18}\left(n_{18 W}+n_{18 A}+n_{18 B}+n_{18 E}\right)+N_{19}\left(1+n_{19 W}+n_{19 A}+n_{19 B}+n_{19 E}\right)+N_{21}\left(1+n_{21 W}+n_{21 A}+n_{21 B}+n_{21 E}\right) \\
& +N_{22}\left(2+n_{22 W}+n_{22 A}+n_{22 B}+n_{22 E}\right)+N_{23}\left(2+n_{23 W}+n_{23 A}+n_{23 B}+n_{23 E}\right)+N_{24}\left(2+n_{24 W}+n_{24 A}+n_{24 B}+n_{24 E}\right) \\
& +N_{25}\left(3+n_{25 W}+n_{25 A}+n_{25 B}+n_{25 E}\right)+N_{26}\left(4+n_{26 W}+n_{26 A}+n_{26 B}+n_{26 E}\right)+N_{27}\left(n_{27 W}+n_{27 A}+n_{27 B}+n_{27 E}\right) \\
& +N_{28}\left(n_{28 \mathrm{~W}}+n_{28 \mathrm{~A}}+n_{28 B}+n_{28 E}\right)+N_{29}\left(n_{29 \mathrm{~W}}+n_{29 \mathrm{~A}}+n_{29 B}+n_{29 E}\right)+N_{31}\left(1+n_{31 \mathrm{~W}}+n_{31 A}+n_{31 B}+n_{31 E}\right) \\
& +N_{32}\left(1+n_{32 W}+n_{32 A}+n_{32 B}+n_{32 E}\right)+N_{33}\left(3+n_{33 W}+n_{33 A}+n_{33 B}+n_{33 E}\right)+N_{34}\left(3+n_{34 W}+n_{34 A}+n_{34 B}+n_{34 E}\right) \\
& +N_{35}\left(n_{35 \mathrm{~W}}+n_{35 \mathrm{~A}}+n_{35 \mathrm{~B}}+n_{35 E}\right)+N_{36}\left(n_{36 \mathrm{~W}}+n_{36 \mathrm{~A}}+n_{36 \mathrm{~B}}+n_{36 E}\right)+N_{37}\left(n_{37 \mathrm{~W}}+n_{37 \mathrm{~A}}+n_{37 \mathrm{~B}}+n_{37 E}\right) \\
& +N_{38}\left(n_{38 \mathrm{~W}}+n_{38 \mathrm{~A}}+n_{38 \mathrm{~B}}+n_{38 \mathrm{E}}\right)+N_{39}\left(n_{39 \mathrm{~W}}+n_{39 \mathrm{~A}}+n_{39 B}+n_{39 E}\right)+N_{41}\left(3+n_{41 \mathrm{~W}}+n_{41 \mathrm{~A}}+n_{41 \mathrm{~B}}+n_{41 E}\right) \\
& +N_{42}\left(3+n_{42 W}+n_{42 A}+n_{42 B}+n_{42 E}\right)+N_{43}\left(3+n_{43 W}+n_{43 A}+n_{43 B}+n_{43 E}\right) \\
& +N_{44}+N_{45}\left(1+n_{45 W}+n_{45 A}+n_{45 B}+n_{45 E}\right)+N_{46}\left(1+n_{46 W}+n_{46 \mathrm{~A}}+n_{46 B}+n_{46 E}\right) \\
& +N_{47}+N_{48}\left(1+n_{48 \mathrm{~W}}+n_{48 \mathrm{~A}}+n_{48 B}+n_{48 E}\right)+N_{49}\left(1+n_{49 \mathrm{~W}}+n_{49 \mathrm{~A}}+n_{49 B}+n_{49 E}\right)+N_{51} \\
& =3 N_{01}+2 N_{03}+N_{04}+N_{05}+N_{06}+2 N_{09}+N_{011}+N_{012}+N_{013}
\end{aligned}
$$

From (1) and (2) we obtain

- $2 \cdot f(\mathrm{O})-f(\mathrm{H})$

$$
\begin{aligned}
& N_{2}\left(-1-2 n_{2 A}-4 n_{2 B}-4 n_{2 E}-3 n_{2 F}\right)+N_{3}\left(1-2 n_{3 A}-4 n_{3 B}-4 n_{3 E}-3 n_{3 F}\right)+N_{4}\left(-2 n_{4 A}-4 n_{4 B}-4 n_{4 E}-3 n_{4 F}\right) \\
& +N_{5}\left(-2 n_{5 A}-4 n_{5 B}-4 n_{5 E}-3 n_{5 F}\right)+N_{6}\left(-2 n_{6 A}-4 n_{6 B}-4 n_{6 E}-3 n_{6 F}\right)+N_{7}\left(-2 n_{7 A}-4 n_{7 B}-4 n_{7 E}-3 n_{7 F}\right) \\
& +N_{8}\left(-2 n_{8 A}-4 n_{8 B}-4 n_{8 E}-3 n_{8 F}\right)+N_{9}\left(1-2 n_{9 A}-4 n_{9 B}-4 n_{9 E}-3 n_{9 F}\right)+N_{11}\left(2-2 n_{11 A}-4 n_{11 B}-4 n_{11 E}-3 n_{11 F}\right) \\
& +N_{12}\left(5-2 n_{12 A}-4 n_{12 B}-4 n_{12 E}-3 n_{12 F}\right)+N_{13}\left(6-2 n_{13 A}-4 n_{13 B}-4 n_{13 E}-3 n_{13 F}\right) \\
& +N_{14}\left(7-2 n_{14 A}-4 n_{14 B}-4 n_{14 E}-3 n_{14 F}\right)+N_{15}\left(8-2 n_{15 A}-4 n_{15 B}-4 n_{15 E}-3 n_{15 F}\right) \\
& +N_{16}\left(9-2 n_{16 A}-4 n_{16 B}-4 n_{16 E}-3 n_{16 F}\right)+N_{17}\left(-2 n_{17 A}-4 n_{17 B}-4 n_{17 E}-3 n_{17 F}\right) \\
& +N_{18}\left(-2 n_{18 A}-4 n_{18 B}-4 n_{18 E}-3 n_{18 F}\right)+N_{19}\left(1-2 n_{19 A}-4 n_{19 B}-4 n_{19 E}-3 n_{19 F}\right) \\
& +N_{21}\left(2-2 n_{21 A}-4 n_{21 B}-4 n_{21 E}-3 n_{21 F}\right)+N_{22}\left(3-2 n_{22 A}-4 n_{22 B}-4 n_{22 E}-3 n_{22 F}\right) \\
& +N_{23}\left(4-2 n_{23 A}-4 n_{23 B}-4 n_{23 E}-3 n_{23 F}\right)+N_{24}\left(4-2 n_{24 A}-4 n_{24 B}-4 n_{24 E}-3 n_{24 F}\right) \\
& +N_{25}\left(6-2 n_{25 A}-4 n_{25 B}-4 n_{25 E}-3 n_{25 F}\right)+N_{26}\left(8-2 n_{26 A}-4 n_{26 B}-4 n_{26 E}-3 n_{26 F}\right) \\
& +N_{27}\left(-2 n_{27 A}-4 n_{27 B}-4 n_{27 E}-3 n_{27 F}\right)+N_{28}\left(-2 n_{28 A}-4 n_{28 B}-4 n_{28 E}-3 n_{28 F}\right) \\
& +N_{29}\left(-2 n_{29 A}-4 n_{29 B}-4 n_{29 E}-3 n_{29 F}\right)+N_{31}\left(1-2 n_{31 A}-4 n_{31 B}-4 n_{31 E}-3 n_{31 F}\right) \\
& +N_{32}\left(2-2 n_{32 A}-4 n_{32 B}-4 n_{32 E}-3 n_{32 F}\right)+N_{33}\left(5-2 n_{33 A}-4 n_{33 B}-4 n_{33 E}-3 n_{33 F}\right) \\
& +N_{34}\left(6-2 n_{34 A}-4 n_{34 B}-4 n_{34 E}-3 n_{34 F}\right)+N_{35}\left(-2 n_{35 A}-4 n_{35 B}-4 n_{35 E}-3 n_{35 F}\right) \\
& +N_{36}\left(-2 n_{36 A}-4 n_{36 B}-4 n_{36 E}-3 n_{36 F}\right)+N_{37}\left(-2 n_{37 A}-4 n_{37 B}-4 n_{37 E}-3 n_{37 F}\right) \\
& +N_{38}\left(-2 n_{38 \mathrm{~A}}-4 n_{38 B}-4 n_{38 E}-3 n_{38 \mathrm{~F}}\right)+N_{39}\left(-2 n_{39 A}-4 n_{39 B}-4 n_{39 E}-3 n_{39 F}\right) \\
& +N_{41}\left(4-2 n_{41 A}-4 n_{41 B}-4 n_{41 E}-3 n_{41 F}\right)+N_{42}\left(5-2 n_{42 A}-4 n_{42 B}-4 n_{42 E}-3 n_{42 F}\right) \\
& +N_{43}\left(6-2 n_{43 \mathrm{~A}}-4 n_{43 B}-4 n_{43 E}-3 n_{43 F}\right)-2 N_{44}+N_{45}\left(-3-2 n_{45 A}-4 n_{45 B}-4 n_{45 E}-3 n_{45 F}\right) \\
& +N_{46}\left(-1-2 n_{46 \mathrm{~A}}-4 n_{46 \mathrm{~B}}-4 n_{46 \mathrm{E}}-3 n_{46 \mathrm{~F}}\right)-2 N_{47} \\
& +N_{48}\left(-5-2 n_{48 \mathrm{~A}}-4 n_{48 B}-4 n_{48 E}-3 n_{48 F}\right)+N_{49}\left(-3-2 n_{49 A}-4 n_{49 B}-4 n_{49 E}-3 n_{49 F}\right)-4 N_{51}-3 N_{52} \\
& =6 N_{01}-N_{02}+4 N_{03}-2 N_{05}-4 N_{06}+4 N_{09}-2 N_{011}-4 N_{012}-4 N_{013}-3 N_{014}
\end{aligned}
$$

As we see, Equation (3) does not involve the terms $N_{1}, N_{04}$, and $\left\{n_{i W}\right\}$ related to water. To cancel the terms 
involved with A, B, E and F, we add Equation (3) to the core balances (4) - (7): $2 \cdot f\left(\mathrm{CH}_{3} \mathrm{O}\right)(4), 4 \cdot f\left(\mathrm{C}_{2} \mathrm{H}_{5} \mathrm{O}\right)(5)$, $4 \cdot f\left(\left(\mathrm{CH}_{3}\right)_{2} \mathrm{SO}\right)(6), 3 \cdot f\left(\mathrm{CH}_{3} \mathrm{CN}\right)(7)$ and charge balance (8). Further simplification gives addition of the balance for $\mathrm{K}(9)$, and of the core balance $4 \cdot f\left(\mathrm{CO}_{3}\right)(10)$ :

- $2 \cdot f\left(\mathrm{CH}_{3} \mathrm{O}\right)$

$$
\begin{aligned}
& 2 N_{2} n_{2 A}+2 N_{3} n_{3 A}+2 N_{4} n_{4 A}+2 N_{5} n_{5 A}+2 N_{6} n_{6 A}+2 N_{7} n_{7 A}+2 N_{8} n_{8 A}+2 N_{9} n_{9 A}+2 N_{11} n_{11 A}+2 N_{12} n_{12 A} \\
& +2 N_{13} n_{13 A}+2 N_{14} n_{14 A}+2 N_{15} n_{15 A}+2 N_{16} n_{16 A}+2 N_{17} n_{17 A}+2 N_{18} n_{18 A}+2 N_{19} n_{19 A}+2 N_{21} n_{21 A}+2 N_{22} n_{22 A} \\
& +2 N_{23} n_{23 A}+2 N_{24} n_{24 A}+2 N_{25} n_{25 A}+2 N_{26} n_{26 A}+2 N_{27} n_{27 A}+2 N_{28} n_{28 A}+2 N_{29} n_{29 A}+2 N_{31} n_{31 A}+2 N_{32} n_{32 A} \\
& +2 N_{33} n_{33 A}+2 N_{34} n_{34 A}+2 N_{35} n_{35 A}+2 N_{36} n_{36 A}+2 N_{37} n_{37 A}+2 N_{38} n_{38 A}+2 N_{39} n_{39 A}+2 N_{41} n_{41 A}+2 N_{42} n_{42 A} \\
& +2 N_{43} n_{43 A}+2 N_{44}+N_{45}\left(2+2 n_{45 A}\right)+N_{46}\left(2+2 n_{46 A}\right)+2 N_{48} n_{48 A}+2 N_{49} n_{49 A}=2 N_{05}+2 N_{011}
\end{aligned}
$$

- $4 \cdot f\left(\mathrm{C}_{2} \mathrm{H}_{5} \mathrm{O}\right)$

$$
\begin{aligned}
& 4 N_{2} n_{2 B}+4 N_{3} n_{3 B}+4 N_{4} n_{4 B}+4 N_{5} n_{5 B}+4 N_{6} n_{6 B}+4 N_{7} n_{7 B}+4 N_{8} n_{8 B}+4 N_{9} n_{9 B}+4 N_{11} n_{11 B}+4 N_{12} n_{12 B} \\
& +4 N_{13} n_{13 B}+4 N_{14} n_{14 B}+4 N_{15} n_{15 B}+4 N_{16} n_{16 B}+4 N_{17} n_{17 B}+4 N_{18} n_{18 B}+4 N_{19} n_{19 B}+4 N_{21} n_{21 B}+4 N_{22} n_{22 B} \\
& +4 N_{23} n_{23 B}+4 N_{24} n_{24 B}+4 N_{25} n_{25 B}+4 N_{26} n_{26 B}+4 N_{27} n_{27 B}+4 N_{28} n_{28 B}+4 N_{29} n_{29 B}+4 N_{31} n_{31 B}+4 N_{32} n_{32 B} \\
& +4 N_{33} n_{33 B}+4 N_{34} n_{34 B}+4 N_{35} n_{35 B}+4 N_{36} n_{36 B}+4 N_{37} n_{37 B}+4 N_{38} n_{38 B}+4 N_{39} n_{39 B}+4 N_{41} n_{41 B}+4 N_{42} n_{42 B} \\
& +4 N_{43} n_{43 B}+4 N_{45} n_{45 B}+4 N_{46} n_{46 B}+4 N_{47}+N_{48}\left(4+4 n_{48 B}\right)+N_{49}\left(4+4 n_{49 B}\right)=4 N_{012}
\end{aligned}
$$

- $4 \cdot f\left(\left(\mathrm{CH}_{3}\right)_{2} \mathrm{SO}\right)$

$$
\begin{aligned}
& 4 N_{2} n_{2 E}+4 N_{3} n_{3 E}+4 N_{4} n_{4 E}+4 N_{5} n_{5 E}+4 N_{6} n_{6 E}+4 N_{7} n_{7 E}+4 N_{8} n_{8 E}+4 N_{9} n_{9 E}+4 N_{11} n_{11 E}+4 N_{12} n_{12 E} \\
& +4 N_{13} n_{13 E}+4 N_{14} n_{14 E}+4 N_{15} n_{15 E}+4 N_{16} n_{16 E}+4 N_{17} n_{17 E}+4 N_{18} n_{18 E}+4 N_{19} n_{19 E}+4 N_{21} n_{21 E}+4 N_{22} n_{22 E} \\
& +4 N_{23} n_{23 E}+4 N_{24} n_{24 E}+4 N_{25} n_{25 E}+4 N_{26} n_{26 E}+4 N_{27} n_{27 E}+4 N_{28} n_{28 E}+4 N_{29} n_{29 E}+4 N_{31} n_{31 E}+4 N_{32} n_{32 E} \\
& +4 N_{33} n_{33 E}+4 N_{34} n_{34 E}+4 N_{35} n_{35 E}+4 N_{36} n_{36 E}+4 N_{37} n_{37 E}+4 N_{38} n_{38 E}+4 N_{39} n_{39 E}+4 N_{41} n_{41 E}+4 N_{42} n_{42 E} \\
& +4 N_{43} n_{43 E}+4 N_{45} n_{45 E}+4 N_{46} n_{46 E}+4 N_{48} n_{48 E}+4 N_{49} n_{49 E}+4 N_{51}=4 N_{06}+4 N_{013}
\end{aligned}
$$

- $3 \cdot f\left(\mathrm{CH}_{3} \mathrm{CN}\right)$

$$
\begin{aligned}
& 3 N_{2} n_{2 F}+3 N_{3} n_{3 F}+3 N_{4} n_{4 F}+3 N_{5} n_{5 F}+3 N_{6} n_{6 F}+3 N_{7} n_{7 F}+3 N_{8} n_{8 F}+3 N_{9} n_{9 F}+3 N_{11} n_{11 F}+3 N_{12} n_{12 F} \\
& +3 N_{13} n_{13 F}+3 N_{14} n_{14 F}+3 N_{15} n_{15 F}+3 N_{16} n_{16 F}+3 N_{17} n_{17 F}+3 N_{18} n_{18 F}+3 N_{19} n_{19 F}+3 N_{21} n_{21 F}+3 N_{22} n_{22 F} \\
& +3 N_{23} n_{23 F}+3 N_{24} n_{24 F}+3 N_{25} n_{25 F}+3 N_{26} n_{26 F}+3 N_{27} n_{27 F}+3 N_{28} n_{28 F}+3 N_{29} n_{29 F}+3 N_{31} n_{31 F}+3 N_{32} n_{32 F} \\
& +3 N_{33} n_{33 F}+3 N_{34} n_{34 F}+3 N_{35} n_{35 F}+3 N_{36} n_{36 F}+3 N_{37} n_{37 F}+3 N_{38} n_{38 F}+3 N_{39} n_{39 F}+3 N_{41} n_{41 F}+3 N_{42} n_{42 F} \\
& +3 N_{43} n_{43 F}+3 N_{45} n_{45 F}+3 N_{46} n_{46 F}+3 N_{48} n_{48 F}+3 N_{49} n_{49 F}+3 N_{52}=3 N_{014}
\end{aligned}
$$

- Charge balance

$$
\begin{aligned}
& N_{2}-N_{3}+N_{4}-N_{5}-N_{6}-N_{11}-N_{13}-N_{15}-2 N_{16}-N_{17}-N_{21}-N_{23}-N_{25}-N_{26}-N_{27} \\
& -N_{28}-N_{32}-N_{34}-N_{35}-N_{37}-N_{39}-N_{42}-2 N_{43}+N_{45}-N_{46}+N_{48}-N_{49}=0
\end{aligned}
$$

- $f(\mathrm{~K})$

$$
N_{01}+N_{08}=N_{4}
$$

- $4 \cdot f\left(\mathrm{CO}_{3}\right)$

$$
4 N_{03}+4 N_{09}=4 N_{41}+4 N_{42}+4 N_{43}
$$

As the result of this addition, considered as a kind of linear combination [4], we obtain the simplest form of GEB, expressed in terms of numbers of entities:

$$
\begin{aligned}
& -N_{5}-N_{6}+N_{9}+N_{11}+5 N_{12}+5 N_{13}+7 N_{14}+7 N_{15}+7 N_{16}-N_{17}+N_{19}+N_{21}+3 N_{22}+3 N_{23}+4 N_{24} \\
& +N_{25}+7 N_{26}-N_{27}-N_{28}+N_{31}+N_{32}+5 N_{33}+5 N_{34}-N_{35}-N_{37}-N_{39}=5 N_{01}-N_{02}-N_{08}
\end{aligned}
$$

Applying the relations: 


$$
\begin{aligned}
& {\left[X_{i}^{z_{i}}\right]\left(V_{0}+V\right)=10^{3} \cdot N_{i} / N_{A}, C_{0} V_{0}=10^{3} \cdot N_{01} / N_{A}, C_{01} V_{0}=10^{3} \cdot N_{02} / N_{A},} \\
& C V=10^{3} \cdot N_{07} / N_{A}, C_{1} V=10^{3} \cdot N_{08} / N_{A}
\end{aligned}
$$

(where $N_{A}$-Avogadro's constant), from Equations (11), (12) we have

$$
\begin{aligned}
& -\left[\mathrm{I}^{-}\right]-\left[\mathrm{I}_{3}^{-}\right]+[\mathrm{HIO}]+\left[\mathrm{IO}^{-}\right]+5\left[\mathrm{HIO}_{3}\right]+5\left[\mathrm{IO}_{3}^{-}\right]+7\left[\mathrm{H}_{5} \mathrm{IO}_{6}\right]+7\left[\mathrm{H}_{4} \mathrm{IO}_{6}^{-}\right]+7\left[\mathrm{H}_{3} \mathrm{IO}_{6}^{2-}\right] \\
& -\left[\mathrm{Cl}^{-}\right]+[\mathrm{HClO}]+\left[\mathrm{ClO}^{-}\right]+3\left[\mathrm{HClO}_{2}\right]+3\left[\mathrm{ClO}_{2}^{-}\right]+4\left[\mathrm{ClO}_{2}\right]+5\left[\mathrm{ClO}_{3}^{-}\right]+7\left[\mathrm{ClO}_{4}^{-}\right] \\
& -\left[\mathrm{Br}^{-}\right]-\left[\mathrm{Br}_{3}^{-}\right]+[\mathrm{HBrO}]+\left[\mathrm{BrO}^{-}\right]+5\left[\mathrm{HBrO}_{3}\right]+5\left[\mathrm{BrO}_{3}^{-}\right]-\left[\mathrm{I}_{2} \mathrm{Cl}^{-}\right]-\left[\mathrm{ICl}_{2}^{-}\right]-\left[\mathrm{IBr}_{2}^{-}\right] \\
& =\left(5 C_{0} V_{0}-C_{01} V_{0}-C_{1} V\right) /\left(V_{0}+V\right)
\end{aligned}
$$

Elemental balances for electro-active elements ("players”) are as follows:

$f(\mathrm{Br})$

$$
N_{27}+3 N_{28}+2 N_{29}+N_{31}+N_{32}+N_{33}+N_{34}+N_{38}+2 N_{39}=N_{01}
$$

$f(\mathrm{Cl})$

$$
N_{17}+2 N_{18}+N_{19}+N_{21}+N_{22}+N_{23}+N_{24}+N_{25}+N_{26}+N_{35}+N_{36}+2 N_{37}=N_{02}
$$

$f(\mathrm{I})$

$$
\begin{aligned}
& N_{5}+3 N_{6}+2 N_{7}+2 N_{8}+N_{9}+N_{11}+N_{12}+N_{13}+N_{14}+N_{15} \\
& +N_{16}+2 N_{35}+N_{36}+N_{37}+N_{38}+N_{39}=2 N_{07}+N_{08}
\end{aligned}
$$

Multiplying (14) - (16) by atomic numbers: $Z_{\mathrm{Br}}=35, Z_{\mathrm{Cl}}=17, Z_{\mathrm{I}}=53$, for $\mathrm{Br}, \mathrm{Cl}$ and I, respectively, adding them and applying Equation (12), we have:

$$
\begin{aligned}
& Z_{\mathrm{I}}\left[\mathrm{I}^{-}\right]+3 Z_{\mathrm{I}}\left[\mathrm{I}_{3}^{-}\right]+2 Z_{\mathrm{I}}\left(\left[\mathrm{I}_{2}\right]+\left[\mathrm{I}_{2(\mathrm{~s})}\right]\right)+Z_{\mathrm{I}}\left([\mathrm{HIO}]+\left[\mathrm{IO}^{-}\right]\right)+Z_{\mathrm{I}}\left(\left[\mathrm{HIO}_{3}\right]+\left[\mathrm{IO}_{3}^{-}\right]\right) \\
& +Z_{\mathrm{I}}\left(\left[\mathrm{H}_{5} \mathrm{IO}_{6}\right]+\left[\mathrm{H}_{4} \mathrm{IO}_{6}^{-}\right]+\left[\mathrm{H}_{3} \mathrm{IO}_{6}^{2-}\right]\right)+Z_{\mathrm{Cl}}\left[\mathrm{Cl}^{-}\right]+2 Z_{\mathrm{Cl}}\left[\mathrm{Cl}_{2}\right]+Z_{\mathrm{Cl}}\left([\mathrm{HClO}]+\left[\mathrm{ClO}^{-}\right]\right) \\
& +Z_{\mathrm{Cl}}\left(\left[\mathrm{HClO}_{2}\right]+\left[\mathrm{ClO}_{2}^{-}\right]\right)+Z_{\mathrm{Cl}}\left[\mathrm{ClO}_{2}\right]+Z_{\mathrm{Cl}}\left[\mathrm{ClO}_{3}^{-}\right]+Z_{\mathrm{Cl}}\left[\mathrm{ClO}_{4}^{-}\right] \\
& +Z_{\mathrm{Br}}\left[\mathrm{Br}^{-}\right]+3 Z_{\mathrm{Br}}\left[\mathrm{Br}_{3}^{-}\right]+2 Z_{\mathrm{Br}}\left[\mathrm{Br}_{2}\right]+Z_{\mathrm{Br}}\left([\mathrm{HBrO}]+\left[\mathrm{BrO}^{-}\right]\right)+Z_{\mathrm{Br}}\left(\left[\mathrm{HBrO}_{3}\right]+\left[\mathrm{BrO}_{3}^{-}\right]\right) \\
& +\left(2 Z_{\mathrm{I}}+Z_{\mathrm{Cl}}\right)\left[\mathrm{I}_{2} \mathrm{Cl}^{-}\right]+\left(Z_{\mathrm{I}}+Z_{\mathrm{Cl}}\right)[\mathrm{ICl}]+\left(Z_{\mathrm{I}}+2 Z_{\mathrm{Cl}}\right)\left[\mathrm{ICl}_{2}^{-}\right]+\left(Z_{\mathrm{I}}+Z_{\mathrm{Br}}\right)[\mathrm{IBr}]+\left(Z_{\mathrm{I}}+2 Z_{\mathrm{Br}}\right)\left[\mathrm{IBr}_{2}^{-}\right] \\
& =\left(Z_{\mathrm{Br}} C_{0} V_{0}+Z_{\mathrm{Cl}} C_{01} V_{0}+2 Z_{\mathrm{I}} C V+Z_{\mathrm{I}} C_{1} V\right) /\left(V_{0}+V\right)
\end{aligned}
$$

After subtracting (13) from (17), we get the equation for GEB, identical with one obtained according to Approach I to GEB

$$
\begin{aligned}
& \left(Z_{\mathrm{I}}+1\right)\left[\mathrm{I}^{-}\right]+\left(3 Z_{\mathrm{I}}+1\right)\left[\mathrm{I}_{3}^{-}\right]+2 Z_{\mathrm{I}}\left(\left[\mathrm{I}_{2}\right]+\left[\mathrm{I}_{2(\mathrm{~s})}\right]\right)+\left(\mathrm{Z}_{\mathrm{I}}-1\right)\left([\mathrm{HIO}]+\left[\mathrm{IO}^{-}\right]\right)+\left(\mathrm{Z}_{\mathrm{I}}-5\right)\left(\left[\mathrm{HIO}_{3}\right]+\left[\mathrm{IO}_{3}^{-}\right]\right) \\
& +\left(Z_{\mathrm{I}}-7\right)\left(\left[\mathrm{H}_{5} \mathrm{IO}_{6}\right]+\left[\mathrm{H}_{4} \mathrm{IO}_{6}^{-}\right]+\left[\mathrm{H}_{3} \mathrm{IO}_{6}^{2-}\right]\right)+\left(Z_{\mathrm{Cl}}+1\right)\left[\mathrm{Cl}^{-}\right]+2 \mathrm{Z}_{\mathrm{Cl}}\left[\mathrm{Cl}_{2}\right]+\left(\mathrm{Z}_{\mathrm{Cl}}-1\right)\left([\mathrm{HClO}]+\left[\mathrm{ClO}^{-}\right]\right) \\
& +\left(Z_{\mathrm{Cl}}-3\right)\left(\left[\mathrm{HClO}_{2}\right]+\left[\mathrm{ClO}_{2}^{-}\right]\right)+\left(\mathrm{Z}_{\mathrm{Cl}}-4\right)\left[\mathrm{ClO}_{2}\right]+\left(\mathrm{Z}_{\mathrm{Cl}}-5\right)\left[\mathrm{ClO}_{3}^{-}\right]+\left(\mathrm{Z}_{\mathrm{Cl}}-7\right)\left[\mathrm{ClO}_{4}^{-}\right] \\
& +\left(Z_{\mathrm{Br}}+1\right)\left[\mathrm{Br}^{-}\right]+\left(3 Z_{\mathrm{Br}}+1\right)\left[\mathrm{Br}_{3}^{-}\right]+2 Z_{\mathrm{Br}}\left[\mathrm{Br}_{2}\right]+\left(Z_{\mathrm{Br}}-1\right)\left([\mathrm{HBrO}]+\left[\mathrm{BrO}^{-}\right]\right) \\
& +\left(Z_{\mathrm{Br}}-5\right)\left(\left[\mathrm{HBrO}_{3}\right]+\left[\mathrm{BrO}_{3}^{-}\right]\right) \\
& +\left(2 Z_{\mathrm{I}}+Z_{\mathrm{Cl}}+1\right)\left[\mathrm{I}_{2} \mathrm{Cl}^{-}\right]+\left(Z_{\mathrm{I}}+Z_{\mathrm{Cl}}\right)[\mathrm{ICl}]+\left(Z_{\mathrm{I}}+2 Z_{\mathrm{Cl}}+1\right)\left[\mathrm{ICl}_{2}^{-}\right]+\left(Z_{\mathrm{I}}+Z_{\mathrm{Br}}\right)[\mathrm{IBr}]+\left(Z_{\mathrm{I}}+2 Z_{\mathrm{Br}}+1\right)\left[\mathrm{IBr}_{2}^{-}\right] \\
& =\left(Z_{\mathrm{Br}} C_{0} V_{0}+Z_{\mathrm{Cl}} C_{01} V_{0}+2 Z_{\mathrm{I}} C V+Z_{\mathrm{I}} C_{1} V\right) /\left(V_{0}+V\right)
\end{aligned}
$$

The balance (18) is equivalent to the balance (13).

A remark is needed in relation to the charge balance. Rewriting Equation (8) in terms of concentrations (see 
Equation (12)), we have

$$
\begin{aligned}
& {\left[\mathrm{H}^{+}\right]-\left[\mathrm{OH}^{-}\right]+\left[\mathrm{K}^{+}\right]-\left[\mathrm{I}^{-}\right]-\left[\mathrm{I}_{3}^{-}\right]+\left[\mathrm{IO}^{-}\right]-\left[\mathrm{IO}_{3}^{-}\right]-\left[\mathrm{H}_{4} \mathrm{IO}_{6}^{-}\right]-2\left[\mathrm{H}_{3} \mathrm{IO}_{6}^{2-}\right]-\left[\mathrm{Cl}^{-}\right]-\left[\mathrm{ClO}^{-}\right]} \\
& -\left[\mathrm{ClO}_{2}^{-}\right]-\left[\mathrm{ClO}_{3}^{-}\right]-\left[\mathrm{ClO}_{4}^{-}\right]-\left[\mathrm{Br}^{-}\right]-\left[\mathrm{Br}_{3}^{-}\right]-\left[\mathrm{BrO}^{-}\right]-\left[\mathrm{BrO}_{3}^{-}\right]-\left[\mathrm{I}_{2} \mathrm{Cl}^{-}\right]-\left[\mathrm{ICl}_{2}^{-}\right]-\left[\mathrm{IBr}_{2}^{-}\right] \\
& -\left[\mathrm{HCO}_{3}^{-}\right]-2\left[\mathrm{CO}_{3}^{2-}\right]+\left[\mathrm{CH}_{3} \mathrm{OH}_{2}^{+}\right]-\left[\mathrm{CH}_{3} \mathrm{O}^{-}\right]+\left[\mathrm{C}_{2} \mathrm{H}_{5} \mathrm{OH}_{2}^{+}\right]-\left[\mathrm{C}_{2} \mathrm{H}_{5} \mathrm{O}^{-}\right]=0
\end{aligned}
$$

As we see, Equation (19) involves the ionic species related to amphiprotic co-solvents. However, in accordance with the remarks presented in [5], the solvates of pairs of ions: $\left(\mathrm{CH}_{3} \mathrm{OH}_{2}^{+}, \mathrm{CH}_{3} \mathrm{O}^{-}\right)$and $\left(\mathrm{C}_{2} \mathrm{H}_{5} \mathrm{OH}_{2}^{+}\right.$, $\mathrm{C}_{2} \mathrm{H}_{5} \mathrm{O}^{-}$) can be perceived as pairs of solvates of $\mathrm{H}^{+}$and $\mathrm{OH}^{-}$ions.

\section{Final Comments}

The complex redox system in a mixture with five solvents is considered. The discussion can be extended on mixtures with $S$ solvents, $A_{1}, \cdots, A_{S}$, where at least one of the co-solvents has amphiprotic properties. In such systems, the solvates $X_{i}^{z_{i}} \cdot n_{i A_{1}} \cdots n_{i A_{S}}$ are considered/admitted, where $n_{i A_{j}} \geq 0 \quad(j=1, \cdots, S)$; Equation (13) is the simplest/shortest form of GEB obtained for the redox system considered according to Approach II to GEB.

Equation (13) was obtained from linear combination of the related balance $2 \cdot f(\mathrm{O})-f(\mathrm{H})$ (Equation (3)) with: charge balance (Equation (8)), elemental balances for other "fans" (C, K) (Equations (9), (10)), and core balances (Equations (4) - (7)) related to organic solvents in this system. This GEB does not involve the species composed only of "fans": H, O, C, K. In particular, it does not contain the components explicitly related to the solvent species. The paper is an illustration of the compact formulation of redox systems according to GATES/ GEB Principles, presented in Ref. [5].

One can notice that uncharged (neutral) species: $\mathrm{I}_{2}, \mathrm{I}_{2(\mathrm{~s})}, \mathrm{Cl}_{2}, \mathrm{Br}_{2}, \mathrm{ICl}, \mathrm{IBr}$ are not present in Equation (13). Note also that $\mathrm{Cl}^{-}, \mathrm{Br}^{-}, \mathrm{I}^{-}, \mathrm{Cl}_{2}, \mathrm{Br}_{2}, \mathrm{I}_{2}, \mathrm{I}_{2(\mathrm{~s})}, \mathrm{Br}_{3}^{-}, \mathrm{I}_{3}^{-}, \mathrm{I}_{2} \mathrm{Cl}^{-}, \mathrm{ICl}, \mathrm{ICl}_{2}^{-}, \mathrm{IBr}$, and $\mathrm{IBr}_{2}^{-}$are the species composed only of "players". In other species, "players" are associated with "fans", e.g. in $\mathrm{HIO}_{3}$ (I-“player"; $\mathrm{H}$, $\mathrm{O}-$ “fans").

Note that—at the start—-the Approach II does not distinguish between "fans" and "players"; the terms "fans" and "players" are used here only for the needs of the Approach I to GEB. In further parts of this text, the "players" (the electro-active elements) are distinguished later only to indicate the equivalency of the Approaches I and II.

\section{References}

[1] http://quoteinvestigator.com/2011/05/13/einstein-simple/

[2] Michałowski, T., Toporek, M., Michałowska-Kaczmarczyk, A.M. and Asuero, A.G. (2013) New Trends in Studies on Electrolytic Redox Systems. Electrochimica Acta, 109, 519-531. http://dx.doi.org/10.1016/j.electacta.2013.07.125

[3] Michałowski, T., Michałowska-Kaczmarczyk, A.M. and Toporek, M. (2013) Formulation of General Criterion Distinguishing between Non-Redox and Redox Systems, Electrochimica Acta, 112, 199-211. http://dx.doi.org/10.1016/j.electacta.2013.08.153

[4] Michałowska-Kaczmarczyk, A.M. and Michałowski, T. (2013) Comparative Balancing of Non-Redox and Redox Electrolytic Systems and Its Consequences. American Journal of Analytical Chemistry, 4, 46-53. http://dx.doi.org/10.4236/ajac.2013.410A1006

[5] Michałowska-Kaczmarczyk, A.M. and Michałowski, T. (2014) Compact Formulation of Redox Systems According to GATES/GEB Principles, Journal of Analytical Sciences, Methods and Instrumentation, 4, 39-45. http://dx.doi.org/10.4236/jasmi.2014.42006

[6] Michałowski, T., Ponikvar-Svet, M., Asuero, A.G. and Kupiec, K. (2012) Thermodynamic and Kinetic Effects Involved with $\mathrm{pH}$ Titration of As(III) with Iodine in a Buffered Malonate System. Journal of Solution Chemistry, 41, 436-446. http://dx.doi.org/10.1007/s10953-012-9815-6

[7] Michałowski, T. (2011) Application of GATES and MATLAB for Resolution of Equilibrium, Metastable and NonEquilibrium Electrolytic Systems, Chapter 1. In: Michałowski, T., Ed., Applications of MATLAB in Science and Engineering, InTech-Open Access Publisher in the Fields of Science, Technology and Medicine, 1-34. http://www.intechopen.com/books/show/title/applications-of-matlab-in-science-and-engineering

[8] Michałowska-Kaczmarczyk, A.M., Michałowski, T., Asuero, A.G., Rymanowski, M. and Toporek, M. (2014) Formu- 
lation of Titration Curves for Some Redox Systems. American Journal of Analytical Chemistry, 5, 861-878. http://dx.doi.org/10.4236/ajac.2014.513095

[9] Pilarski, B., Dobkowska, A., Foks, H. and Michałowski, T. (2010) Modeling of Acid-Base Equilibria in Binary-Solvent Systems: A Comparative Study. Talanta, 80, 1073-1080. http://dx.doi.org/10.1016/j.talanta.2009.07.033

[10] Asuero, A.G., Pilarski, B., Dobkowska, A. and Michałowski, T. (2013) On the Isohydricity Concept-Some Comments, Talanta 112, 49-54. http://dx.doi.org/10.1016/j.talanta.2013.03.067

[11] Michałowski, T., Pilarski, B., Asuero, A.G. and Michałowska-Kaczmarczyk, A.M. (2014) Modeling of Acid-Base Properties in Binary-Solvent Systems, Chapter 9.4. In: Wypych. G., Ed., Handbook of Solvents, Vol. 1 Properties, ChemTec Publishing, Toronto, 623-648. 
Scientific Research Publishing (SCIRP) is one of the largest Open Access journal publishers. It is currently publishing more than 200 open access, online, peer-reviewed journals covering a wide range of academic disciplines. SCIRP serves the worldwide academic communities and contributes to the progress and application of science with its publication.

Other selected journals from SCIRP are listed as below. Submit your manuscript to us via either submit@scirp.org or Online Submission Portal.
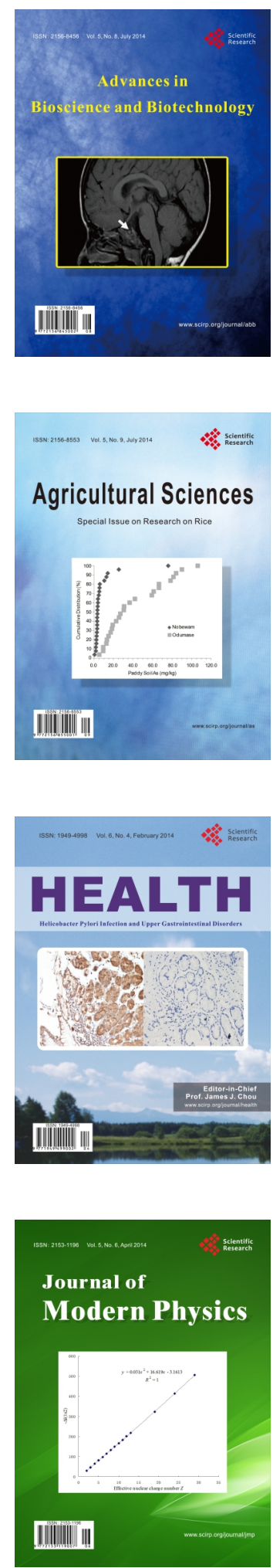
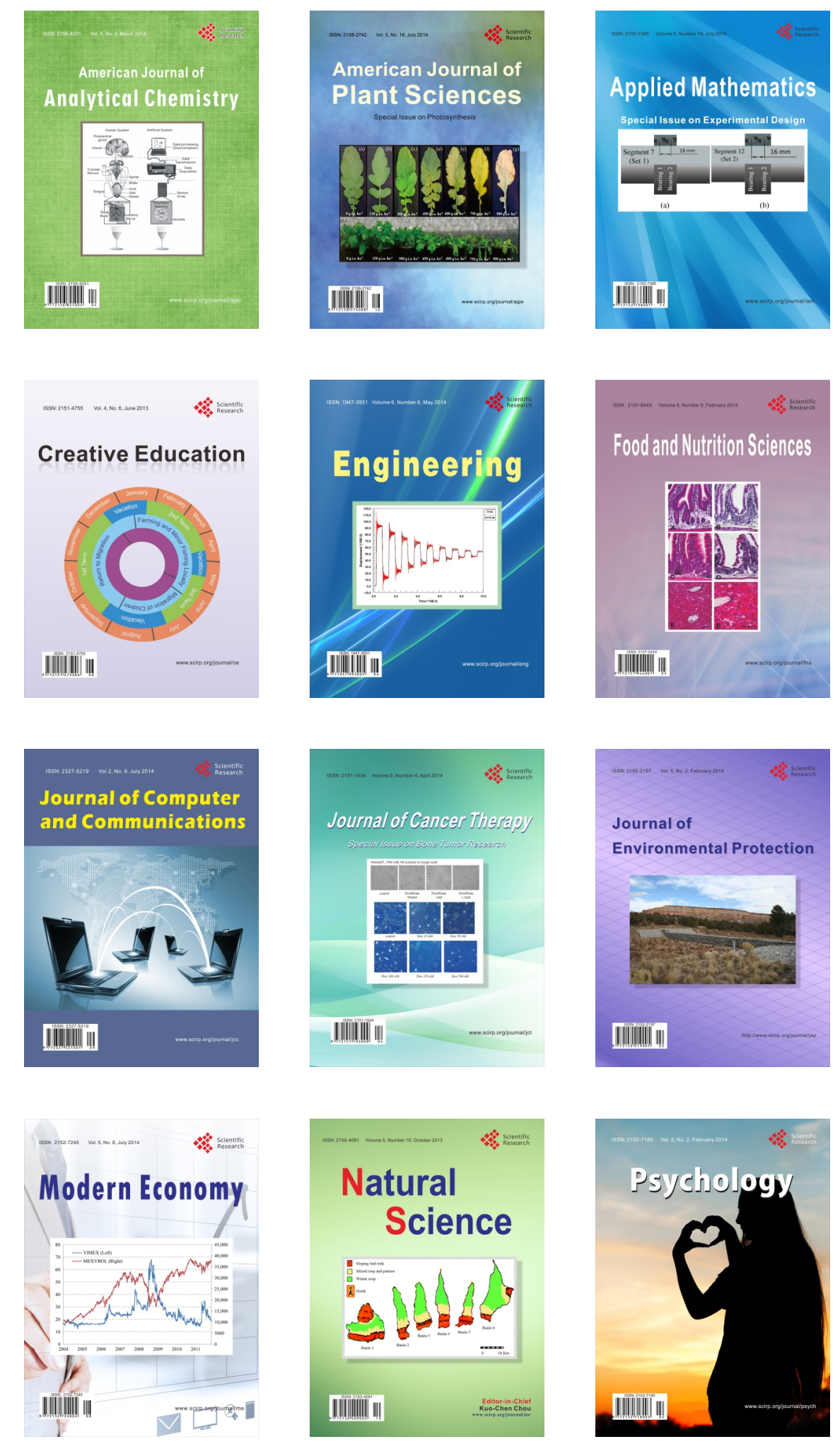